\title{
ÖGIM Fortbildungsveranstaltungen 2014
}

\section{ÖGIM Jahrestagung, 25.-27. September 2014}

Zusätzlich zu den Tandem- und Gesellschaftssitzungen bietet die ÖGIM im Rahmen der Jahrestagung Handson-Kurse und eine Fortbildungsschiene an. Nur buchbar in Kombination mit einer Anmeldung zum Gesamtkongress. Weitere Informationen finden Sie unter: www.oegim.at/aktuell/jahrestagung

Hands-on-Kurse:

Gastroskopie

Bronchoskopie

Duplexsonographie Carotis

Reanimation/Intubation

Spirometrie

Duplexsonographie Venen

Echokardiographie

ABI Messung inklusive oszillometrische Methoden

Fortbildungsschiene:

Nephrologie

Endokrinologie

Kardiologie

Hämatologie und Hämastaseologie

Geriatrie und Palliativmedizin

Gastroenterologie

Infektionskrankheiten

Rheumatologie

Hepatologie

Pneumologie

Laden Sie auch die ÖGIM App herunter - kostenlos im App Store und in Google Play!

Hier finden Sie die aktuellen ÖGIM Fortbildungsveranstaltungen mit Evaluierungsmöglichkeit per Knopfdruck!

\section{FAP-Vorbereitungsseminar in Krems}

Seit vielen Jahren bietet die ÖGIM in Krems dreimal pro Jahr Seminare zur Vorbereitung auf die Facharztprüfung an. Die Seminare sind als Multiple-Choice-Vorträge aufgebaut, es kommt bei der interaktiven Kommunikation mit den Vortragenden ein Digivote-System zum Einsatz.

Nächster Termin: 21.-23. August 2014

Anmeldung erforderlich, Informationen unter: www.oegim.at/aus-und-fortbildung/facharztpruefung/ seminare

\section{FLIP - Fortbildung Live Internet Print}

Die ÖGIM präsentiert mit FLIP - Fortbildung///Live/ Internet/Print ein neues Fortbildungsformat, das Ihnen den Erwerb von insgesamt 72 Fortbildungspunkten über diese drei Kanäle ermöglicht. Bei den kostenlosen Veranstaltungen bieten wir Ihnen ein Digivote-System zur aktiven Teilnahme an.

Wir freuen uns, zahlreiche Teilnehmer bei den Veranstaltungen begrüßen zu dürfen und bitten um Anmeldung unter www.oegim-flip.at

Termine 2014:

15. September - Schilddrüse und Nebenschilddrüse

20. Oktober - Gelenke

24. November - Lymphknoten 